\title{
DUAL-BAND DUAL-POLARIZED ARRAY FOR WLAN APPLICATIONS
}

\author{
J. M. Steyn, J. W. Odendaal, and J. Joubert \\ Centre for Electromagnetism \\ Department of Electrical, Electronic and Computer Engineering \\ University of Pretoria \\ Pretoria 0002, South Africa
}

\begin{abstract}
This paper presents a dual-band dual-polarized antenna array design for WLAN applications. Four double-dipole elements are orthogonally interleaved to facilitate operation in both the standard WLAN frequency bands (IEEE 802.11b and IEEE 802.11a) simultaneously. The two linear polarizations have separate ports. The presented design is characterized by dual-band operation, reasonably good front-to-back ratios, average gains of $5.2 \mathrm{dBi}$ and $6.2 \mathrm{dBi}$ over the 2.4 and $5.2 \mathrm{GHz}$ bands respectively, stable end-fire radiation patterns and very low cross-polarization levels.
\end{abstract}

\section{INTRODUCTION}

The recent growth in the ambit of modern wireless communication has increased the demand for multi-band antennas that can satisfy the requirements pertaining to WLANs (Wireless Local Area Networks). Dual-band antennas that can cover both the $2.4 \mathrm{GHz}(2.4-2.484 \mathrm{GHz})$ band and the $5.2 \mathrm{GHz}(5.15-5.85 \mathrm{GHz})$ band for the IEEE $802.11 \mathrm{~b}$ and IEEE 802.11a WLAN standards respectively are thus in demand. A second requirement for some WLAN applications may be polarization diversity, which can be achieved by making use of dual-polarized arrays similar to those shown in [1]. The need for data rates higher than $54 \mathrm{Mbps}$ and thus higher bandwidth efficiency [2] for WLAN systems have increased MIMO (Multiple-Input-MultipleOutput) related research [3]. MIMO systems not only use multipath propagation in a constructive way, but increase the robustness and capacity of the whole system. Polarization diversity or dual-polarized configurations can assist in realizing bandwidth efficient schemes such

Corresponding author: J. Joubert (jjoubert@postino.up.ac.za). 
as the MIMO-OFDM (Multiple Input Multiple Output-Orthogonal Frequency Division Multiplexing) scheme, by increasing the system capacity without adding additional antennas at the receiver and transmitter. Various adaptive modulation and demodulation methods have been proposed in conjunction with the OFDM scheme [2] to provide a potentially more efficient solution.

It is relatively easy to realize a dual-polarized structure by making use of two ports in conjunction with a circular, square or annular microstrip antenna [4], but it is more challenging to design a structure that has the capacity to support dual-band and dualpolarized operation. A fair amount of research has been done to develop suitable antenna elements with the capacity to support orthogonal polarizations with dual-frequency bands. Dual-frequency elements such as stacked-, notched- and dichroic patches have also been considered to be modified to facilitate dual-polarized operation. The size of the elements, the high cross-polarization levels associated with dichroic- and stacked patches and the complex routing of feeding networks needed to implement some of the antenna patch designs [4] disqualifies these options in general to be used in dual-polarized arrays.

Printed dipoles and slot antennas, which occupy less space, have also been investigated and the feasibility of some of these options for SAR applications was ascertained in [5]. The printed bow-tie design presented in [1] makes use of four dipoles and four ports to realize a very wide bandwidth dual-polarized design for $\mathrm{C}$ - and $\mathrm{X}$-band applications. Two different dual-polarized configurations were simulated, but not verified by means of physical measurements. Another attractive printed planar design is the double Rhombus antenna presented in [6] - proposed for ultra-wideband phased array systems. This structure has the potential capacity to support dual-band operation and also exhibits other favorable characteristics such as end-fire radiation patterns, light weight, small dimensions and low manufacturing costs.

This paper presents a dual-band dual-polarized (DBDP) antenna design for WLAN applications. The antenna is in the form of an array consisting of four double-dipole radiators. The basic radiating element consists of a rhombus shaped dipole above a planar ground plane for operation in the lower WLAN frequency band and a rectangular dipole with added director for operation in the higher WLAN frequency band. Dual-linear polarization is achieved by orthogonally combining two two-element subarrays. If mounted on a wall the one two-element subarray will radiate horizontal linear polarization and the other vertical linear polarization. Each polarization is fed by a separate port. 


\section{ANTENNA CONFIGURATION}

\subsection{Single Element}

The single-element design has been described in [7] and is shown in Fig. 1. It consists of two parallel dipoles of different shapes, fed by a microstrip line. The lengths $\left(L_{3}\right.$ and $\left.L_{5}\right)$ of the dipoles are the key parameters responsible for the two different operating frequencies. The combined length $L_{1}+L_{2}$ is the spacing between the planar ground and the lower band dipole. The length $L_{4}$ represents the spacing between the lower band and the higher band dipole and the position, width and length of the passive director are defined by $d, W_{d}$ and $L_{d}$ respectively. The two halves of the dipoles are printed on either side of the substrate. The antenna was designed to operate over the $2.4 / 5.2 \mathrm{GHz}$ bands on a Rogers RO4003C substrate with a dielectric constant of 3.38, a height of $0.813 \mathrm{~mm}$ and a loss tangent $(\tan \delta)$ of 0.0027 . The single-element dimensions are summarized in Tables 1 and 2.

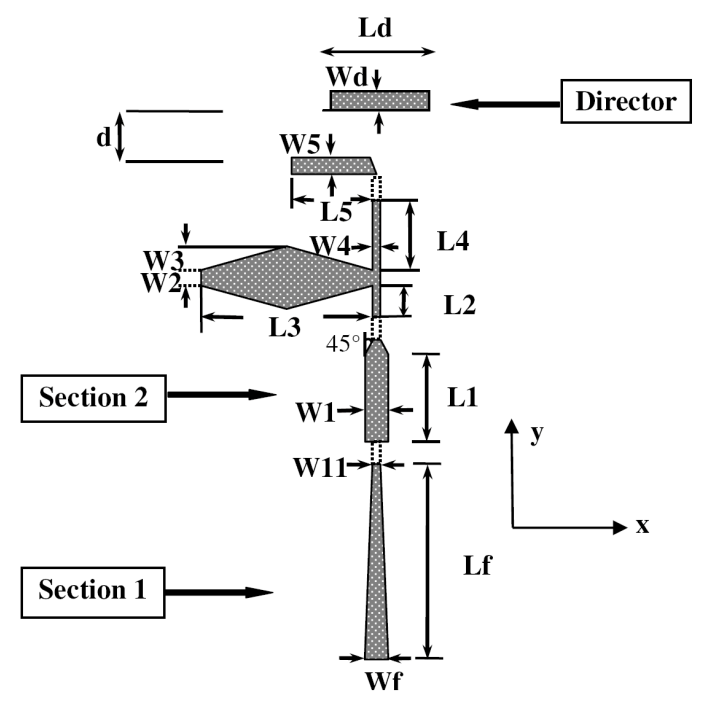

Figure 1. The upper layer of the single-element showing the design parameters.

Table 1. The lengths of the single-element configuration [units: $\mathrm{mm}$ ].

\begin{tabular}{cccccccc}
\hline$L_{1}$ & $L_{2}$ & $L_{3}$ & $L_{4}$ & $L_{5}$ & $L_{f}$ & $L_{d}$ & $d$ \\
19.0 & 4.3 & 25.0 & 3.0 & 9.5 & 39.0 & 17.0 & 3.5 \\
\hline
\end{tabular}


Table 2. The widths of the single-element configuration [units: $\mathrm{mm}$ ].

\begin{tabular}{cccccccc}
$W_{1}$ & $W_{11}$ & $W_{2}$ & $W_{3}$ & $W_{4}$ & $W_{5}$ & $W_{f}$ & $W_{d}$ \\
1.4 & 0.7 & 4.2 & 2.5 & 0.6 & 2.5 & 1.4 & 1.0 \\
\hline
\end{tabular}

Table 3. The feed dimensions [units: $\mathrm{mm}$ ].

\begin{tabular}{c|c|c|c|c|c|c|c|c|c|c}
\hline$L_{s}$ & $L_{e}$ & $L_{t 1}$ & $L_{t 2}$ & $L_{h}$ & $L_{u 1}$ & $L_{u 2}$ & $L_{v 1}$ & $L_{v 2}$ & $W_{e}$ & $W_{u}$ \\
44.3 & 8.9 & 6.6 & 18.6 & 37.8 & 6.8 & 11.3 & 37.7 & 25.7 & 4.6 & 3.7 \\
\hline
\end{tabular}
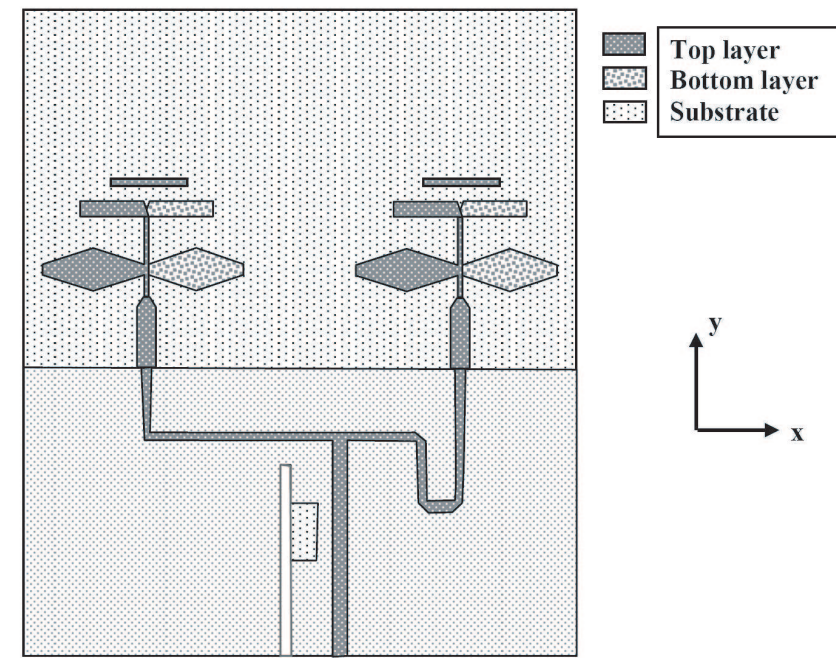

Figure 2. The 1st of the two-element configuration.

\subsection{Two- and Four-element Configurations}

Two different two-element configurations were assembled by combining four of the single-elements. This was done to (i) increase the gain of the antenna, and (ii) to facilitate the transformation into a dualpolarized array, similar to the dual-polarized dipole designs suggested in [1], but in this letter with only one $50 \Omega$ port for each polarization. Fig. 2 shows the 1st of the two-element subarray configurations (for horizontal polarization). Extra care was taken to avoid interference between the ground planes and the respective feedlines. Substrate cuts and different heights for the horizontal feedlines were necessary to achieve the latter. The 2nd configuration (for vertical polarization) has a slightly different feeding network layout — the design parameters 
of both feed configurations given in Fig. 3. The 2 nd configuration was implemented with a slit from above. The feed dimensions for both configurations are summarized in Table 3. The array configuration was constructed via the orthogonal interleaving of the two two-element subarrays. The distance between the centres of the two co-polarized single-elements was chosen to be equal to $58 \mathrm{~mm}$. The distance is approximately equal to $0.44 \lambda_{0}$ at $2.3 \mathrm{GHz}$, the lower limit of the first frequency band and equal to $1.13 \lambda_{0}$ at $5.85 \mathrm{GHz}$, the upper frequency limit of the $5.2 \mathrm{GHz}$ WLAN frequency band. The total structure occupies a volume of $148 \mathrm{~mm} \times 148 \mathrm{~mm} \times 101.3 \mathrm{~mm}(L \times W \times H)$. The final array configuration is shown in Fig. 4.

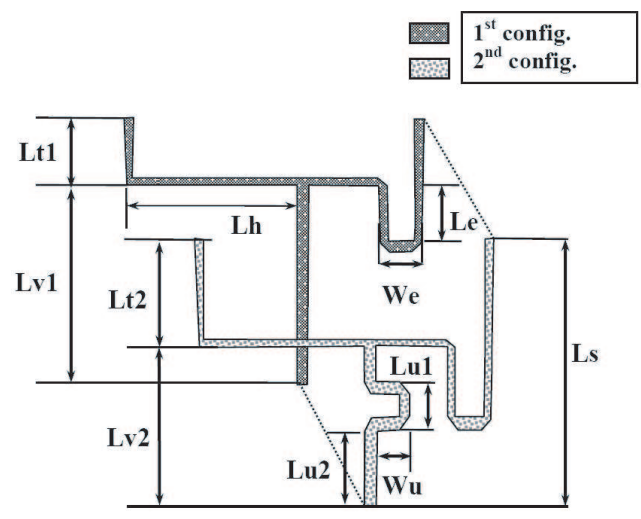

Figure 3. The two feeding configurations showing the design parameters.

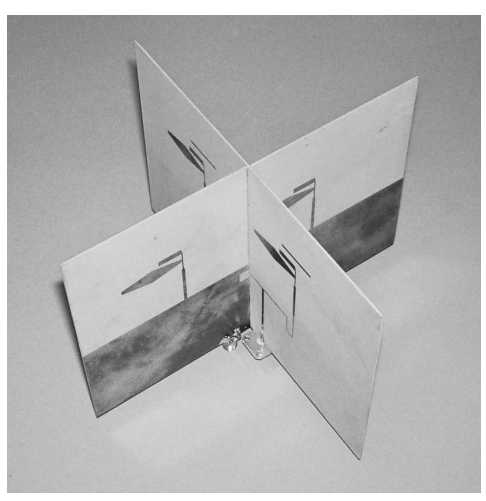

Figure 4. The physical DBDP prototype.

\section{SIMULATION AND MEASURED RESULTS}

All the simulations were conducted with the aid of CST Microwave Studio $\AA$ and the measurements were performed at the Compact Antenna Range of the University of Pretoria.

\subsection{Reflection Coefficient, Coupling and Gain}

The DBDP array configuration was simulated and also measured in terms of port and radiation characteristics. The horizontally polarized array, excited at Port 1, delivered measured impedance bandwidths $(2: 1)$ of $32.5 \%$ and $37.1 \%$ over the $2.4 / 5.2 \mathrm{GHz}$ bands respectively. The vertically polarized array, excited at Port 2 , achieved very similar results with only slightly narrower bandwidths. The measured and 


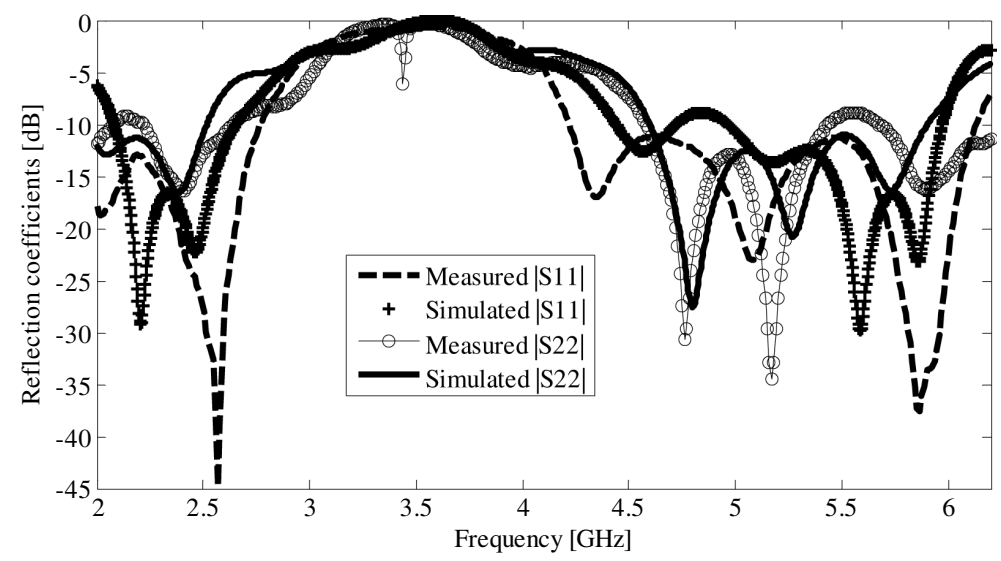

Figure 5. The reflection coefficient levels of the horizontal (Port 1) and vertical (Port 2) polarizations.

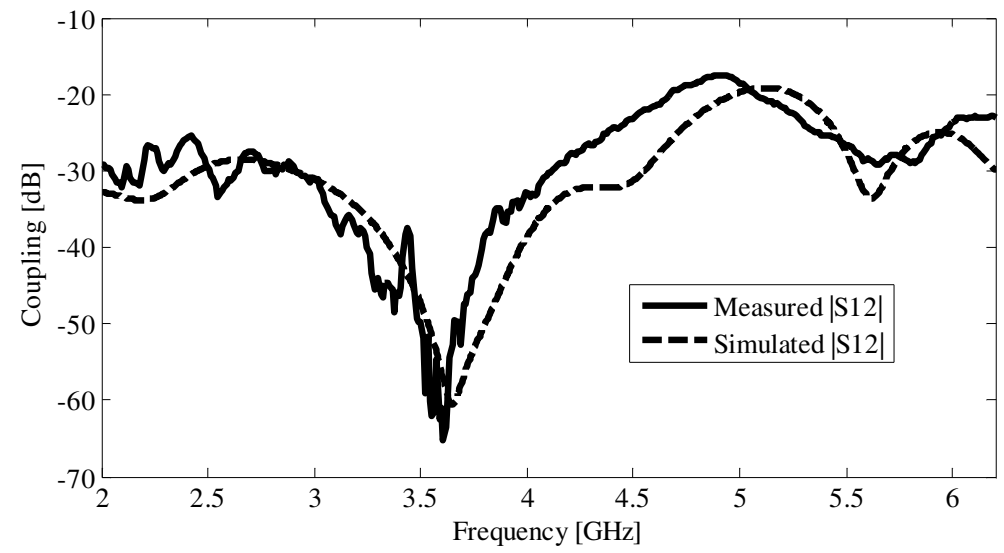

Figure 6. The coupling between Port 1 and Port 2 .

simulated reflection coefficients of both polarizations are shown in Fig. 5. The coupling between the two ports was also simulated and measured, and good correlation between the two sets of data was found, as shown in Fig. 6. The largest in-band coupling (at the low end of the higher band) is approximately $-20 \mathrm{~dB}$. The approximate average simulated bore-sight gains of the two-element subarrays over the lower and upper frequency band are $5.2 \mathrm{dBi}$ and $6.2 \mathrm{dBi}$ respectively (see Figs. 7 and 8) - compared to approximately $4.0 \mathrm{dBi}$ and $4.6 \mathrm{dBi}$ for the single element radiator described in [7]. The somewhat below 
expected improvement in gain can be attributed to a combination of factors, i.e., (i) interaction between the co-polarized elements (the two elements of the array share a common planar ground plane and the interaction between currents will be greater in the lower band where the spacing between the elements is less than half a wavelength), (ii) radiation and feed losses from the feed network, and (iii) asymmetry of the crossed structure. The differences between the simulated and measured gain data (also shown in Figs. 7 and 8) can possibly be attributed to manufacturing inaccuracies and measurement error.

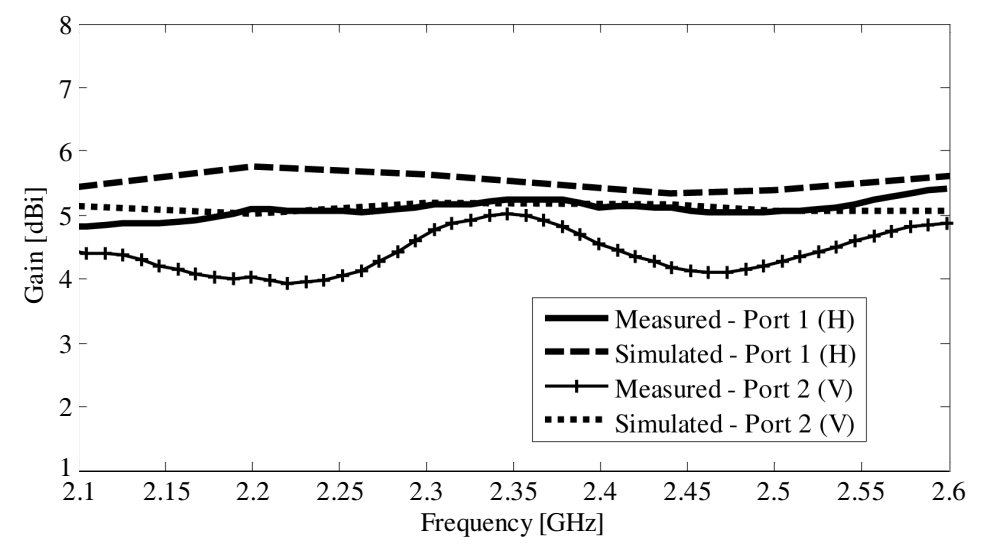

Figure 7. The measured and simulated gain over the $2.4 \mathrm{GHz}$ band.

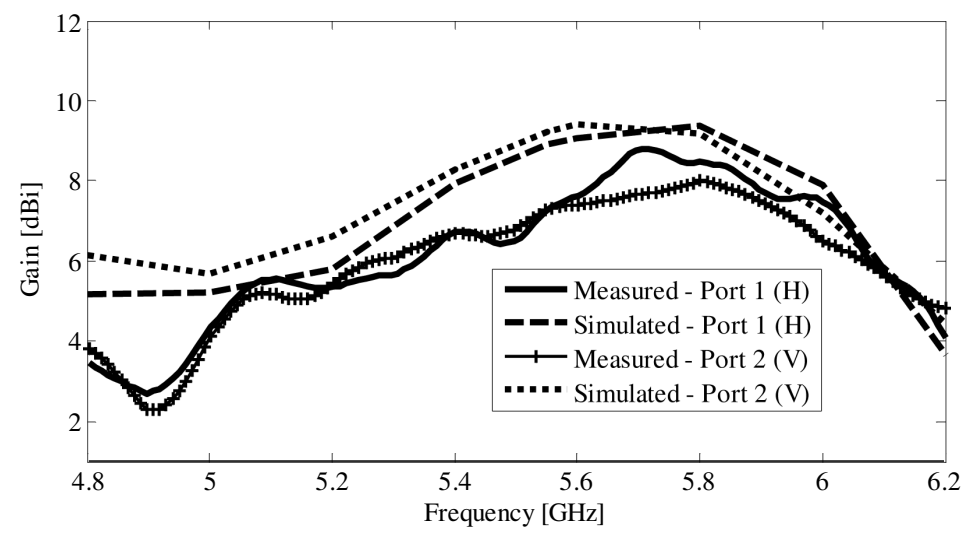

Figure 8. The measured and simulated gain over the $5.2 \mathrm{GHz}$ band. 


\subsection{Radiation Patterns}

Figures 9 and 10 show the normalized radiation patterns of the antenna in the $E$-plane, whereas the $H$-plane radiation patterns are shown in Figs. 11 and 12. Good agreement is observed between the simulated and measured results in both the principle planes. The front-toback ratios pertaining to the horizontally and vertically polarized arrays were very similar to each other, and was approximately $10 \mathrm{~dB}$ in the $2.4 \mathrm{GHz}$ band and approximately $15 \mathrm{~dB}$ in the $5.2 \mathrm{GHz}$ band. Slight discrepancies between the measured $E$ - and $H$-plane back-lobe levels can be attributed to measurement set-up alignment tolerances. The cross-polarization levels were overall very low in the main beam direction - generally lower than $-20 \mathrm{~dB}$ except for the lower band $H$ plane vertical polarization case, which were simulated and measured to be a few decibels higher - possibly due to the difference in the feeding networks of the two subarrays.
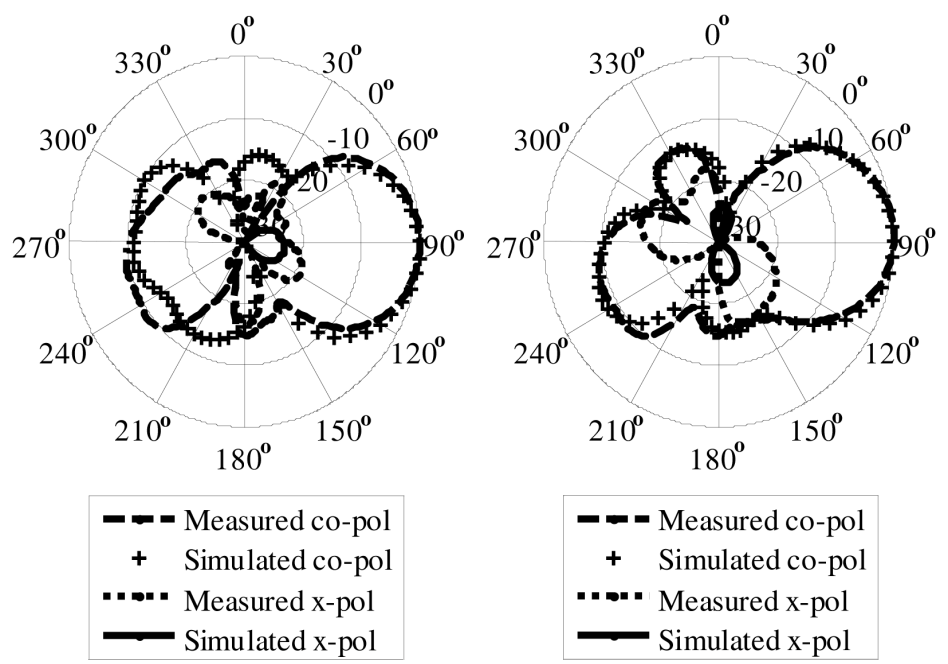

Figure 9. The measured and simulated normalized radiation pattern (as function of $\varphi, \theta=90^{\circ}$, with reference to the coordinate system in Fig. 2) at $2.44 \mathrm{GHz}$ in the $E$-plane. (a) Horizontal polarization. (b) Vertical polarization. 

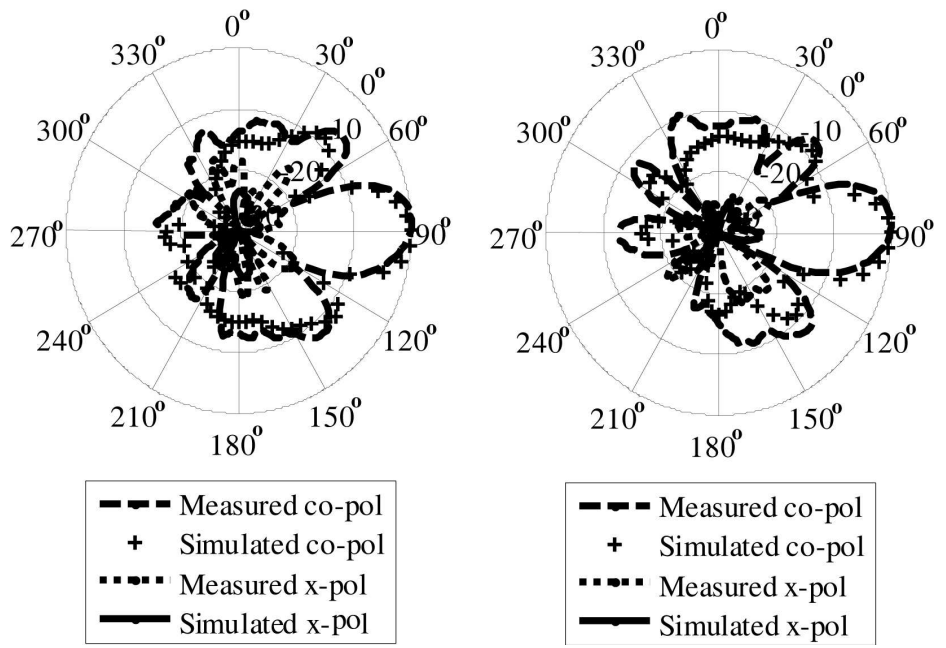

Figure 10. The measured and simulated normalized radiation pattern (as function of $\varphi, \theta=90^{\circ}$, with reference to the coordinate system in Fig. 2) at $5.55 \mathrm{GHz}$ in the $E$-plane. (a) Horizontal polarization. (b) Vertical polarization.
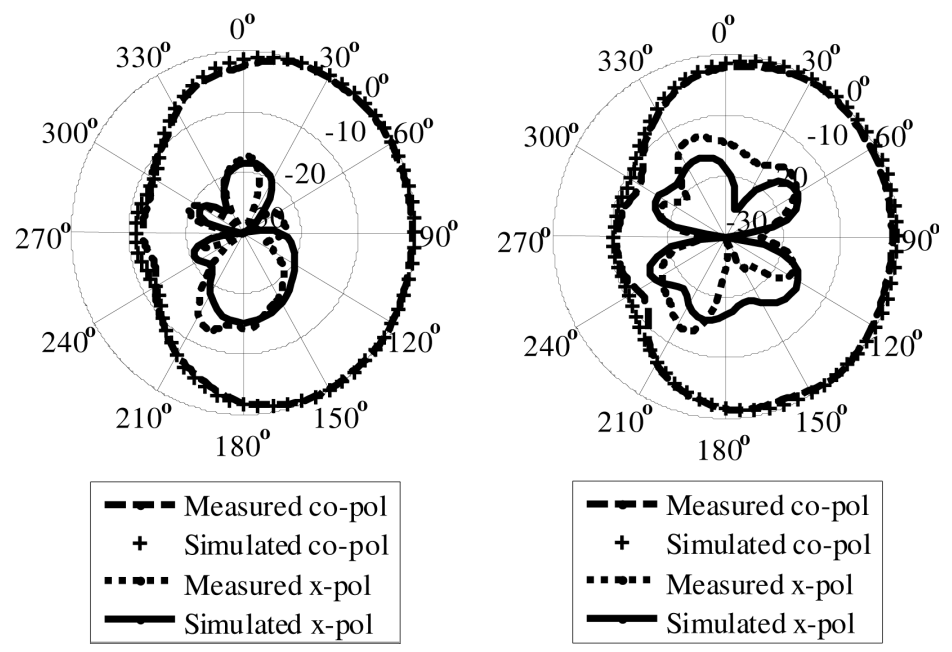

Figure 11. The measured and simulated normalized radiation pattern (as function of $\varphi, \theta=90^{\circ}$, with reference to the coordinate system in Fig. 2) at $2.44 \mathrm{GHz}$ in the $H$-plane. (a) Horizontal polarization. (b) Vertical polarization. 

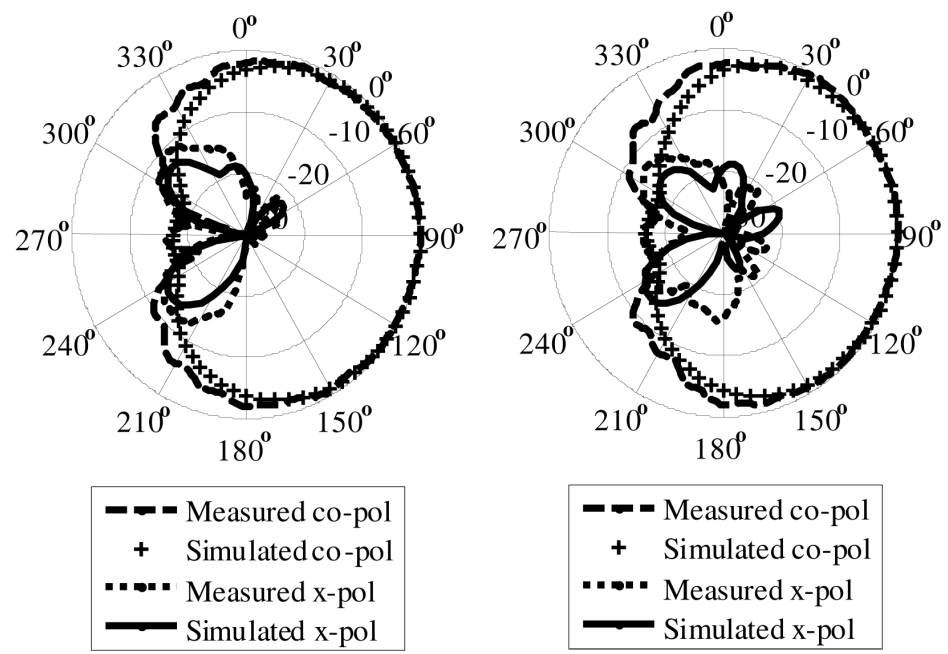

Figure 12. The measured and simulated normalized radiation pattern (as function of $\varphi, \theta=90^{\circ}$, with reference to the coordinate system in Fig. 2) at $5.55 \mathrm{GHz}$ in the $H$-plane. (a) Horizontal polarization. (b) Vertical polarization.

\section{CONCLUSION}

A dual-band dual-polarized array design consisting of four doubledipole radiators is presented as a possible antenna for WLAN applications. Even though the new antenna array is a logical extension of the single dual-band element published in [7], this paper does contain important new information regarding the feeding mechanism of the dual-polarized combination of the four single elements, and interesting new results in terms of coupling between the ports, gain, and radiation patterns. Even though the antenna is not very compact it does exhibit extremely good cross-polarization discrimination, moderate gains in both frequency bands and reasonably good front-to-back ratios, and may be a suitable candidate for dual-polarized dual-band MIMO WLAN systems. The two operating bandwidths more than adequately cover the frequency bands specified by the IEEE 802.11b and IEEE 802.11a WLAN standards, assuming a maximum VSWR of $2: 1$.

\section{REFERENCES}

1. Eldek, A. A., A. Z. Elsherbeni, and C. E. Smith, "Wideband modified printed bow-tie antenna with single and dual 
polarization for C- and X-band applications," IEEE Trans. Antennas Propagat., Vol. 53, No. 9, 3067-3072, 2005.

2. Piechocki, R., P. Fletcher, A. Nix, N. Canagarajah, and J. McGeehan, "A measurement based feasibility study of spacefrequency MIMO detection and decoding techniques for next generation wireless LANS," IEEE Trans. Consumer Electr., Vol. 48, No. 3, 732-737, 2002.

3. Das, N. K., M. Shinozawa, N. Miyadai, T. Taniguchi, and Y. Karasawa, "Experiments on a MIMO system having dual polarization diversity branches," IEICE Trans. Commun., Vol. e89-b, 2522-2529, 2006.

4. Targonski, S. D. and D. M. Pozar, "Dual-band and dual polarized printed antenna element," Elect. Lett., Vol. 34, No. 23, 2193-2194, 1998.

5. Pokuls, R., J. Uher, and D. M. Pozar, "Dual-frequency and dualpolarization microstrip antennas for SAR applications," IEEE Trans. Antennas Propagat., Vol. 46, No. 9, 1289-1296, 1998.

6. Eldek, A. A., "Ultrawideband double rhombus antenna with stable radiation patterns for phased array applications," IEEE Trans. Antennas Propagat., Vol. 55, No. 1, 84-91, 2007.

7. Steyn, J. M., J. W. Odendaal, and J. Joubert, "Double dipole antenna for dual-band WLAN applications," IEEE Microw. Opt. Tech. Lett., Vol. 51, No. 9, 2034-2038, 2009. 